\title{
Dopamine and dopamine receptor D1 associated with decreased social interaction
}

\begin{abstract}
Deficits in social interaction are hallmarks of neurological and psychiatric disorders. However, its underlying mechanism is still unclear. Here, we show that the loss of dendritic cell factor 1 (Dcf1) in the nervous system of mice induces social interaction deficiency, autism-like behaviour, and influences social interaction via the dopamine system. Dopamine receptor D1 agonist rescues this social cognition phenotype, and improves short-term plasticity. Together, this study presents a new genetic mechanism that affects social interaction and may provide a new way to improve positive social interaction and treat autism spectrum disorders.
\end{abstract}

\section{Introduction}

Social interaction is defined as any relationship between two or more individuals. Humans and other primates spend much of their time engaged in social interactions [1], and most of the research is increasingly focused on how people act and understand each other in interactive situations [2, 3]. Positive social interactions contribute to the sense that one's life has meaning [4]. Therefore, the motivation to form and sustain social connections is one of the most powerful, universal, and influential human drives [5]. In fact, lack of social interaction lowers self-esteem and is a bigger health risk factor than smoking and obesity in human beings [6-8]. Thus, it 
is particularly important to study human social interaction. However, it is difficult to develop an experimental paradigm that modulates the quantity of social interactions, making it difficult to understand the neural mechanisms underlying these interactions. Therefore, mice models are good tools to study of the neural mechanism of social behaviour. Autism or autism spectrum disorders is one such defect in social behaviour in humans. They are neurodevelopmental disorders diagnosed based on a triad of criteria, involving deficits in communication and impaired social interaction. Deficits in social interaction are the most recognizable manifestation of autistic behaviours [9]. We have previously shown high expression of Dcf1 (dendritic cell factor 1, also known as TMEM59) in neural stem cells (NSCs) [10]. Dcf1 is a protein consisting of 323 amino acids including an N-terminal signal sequence, a mitochondrial targeting sequential motif, a transmembrane region, and a cytoplasmic tail. Silencing of Dcf1 promotes differentiation of neural stem cells [11], implying its effect on brain neural development. In our previous study, we showed that dendritic spine density, spine head size, dendritic arborization, and postsynaptic density length in hippocampal synapses are significantly decreased in Dcf1 knockout mouse [12]. In this study, we suggest that these changes could be a possible cause for social behavioural disorders. It has been previously shown that social behaviour is closely related to the dendritic spine morphology [13], supporting our initial assumptions.

Dopamine (DA) is a neurotransmitter involved in several neurochemical and neurohormonal actions that affect and modulate animal behaviour and cognition $[14$, 15]. DA and serotonin (5-HT) modulate a variety of brain functions, and their altered 
levels have been implicated in the pathophysiology of affective disorders [16]. DA is associated with motivation, reward, and hedonic states; therefore, increasing DA levels could possibly treat depression [17]. It has been proven that DA agonists can improve depressive symptoms [18]. Dopamine receptor D1 (DRD1) mediates the effects of antidepressant drugs in the forced swim test (FST) whereas, dopamine receptor D2 antagonists blocked the effect of antidepressant drugs in the FST [19, 20]. Although DA and dopaminergic receptors are involved in social interaction, the underlying neural mechanisms are ambiguous. In this study, we aimed to explore the role of Dcf1 in social behaviour using (loss of Dcf1 in the nervous system) NKO mouse.

\section{Results}

\section{NKO mice display dysfunctional social interaction}

The death rate of NKO mice (was higher than wild type (WT) mice from birth to 4 months old, but there was no difference in the sex ratio (Fig. 1A). Furthermore, NKO mice tended to show lesions on the back of the neck (Fig. 1B). No lesions were found in WT mice [9]. As the animals were socially isolated at weaning age we investigated if they were self-inflicted. To explain this, we conducted behavioural experiments including video recording. Videotaping in pre-lesion animals revealed that NKO mice showed an increase in time spent for grooming compared to that in WT controls (Fig. 1C). Thus, excessive grooming and self-injurious behaviour may have caused lesions and excess mortality in NKO mice. Self-grooming in animals is an innate behaviour 
involving hygiene maintenance and social communication [21]. Therefore, we used a modified version of a three-chamber social arena to evaluate animals for their social interaction and ability to discriminate social novelty [9]. NKO mice exhibited a clear preference for interacting with the empty cage rather than with a social partner ('Stranger 1') (Fig. 1D, 1F). Subsequently, a novel social partner ('Stranger 2') was introduced into the previously empty wire cage. WT mice displayed preference for the novel animal, whereas the NKO mice spent more time in the chamber containing a familiar animal (Fig. 1E, 1G), it revealed that losing Dcf1 in the nervous system perturbed the recognition of social novelty. Thus, these results indicate that the NKO mice exhibit autism-relevant behavioural abnormalities.

\section{NKO mice exhibited lower DA and DRD1 levels in the hippocampus}

Neurons in the hippocampus can overdrive DA and 5-HT to distort the emotional change [22, 23]. Thus, we detected the levels of these neurotransmitters in the hippocampus by using HPLC. The retention time of DA and 5-HT was 10 and 11 min, respectively (Supplementary Fig. 1A, 1B). We tested them together, as DA and 5-HT could be separated using a mixed sample of DA and 5-HT standards (Supplementary Fig. 1C). Results revealed that DA concentration in the NKO mice was lower than the WT mice (Fig. 2A, 2B), whereas, 5-HT showed no significant difference in the mouse strains (Fig. 2A, 2C). We also used western blotting to detect the expression of DRD1 in the hippocampus which was also reduced similar to DA (Fig. 2D, 2E). These results show a deficit of DA system in the hippocampus of NKO mice. 
Hippocampus is positioned to modulate the amplitude of the phasic DA signal which is consistent with the proposed behavioural function of the hippocampus [24, 25]. Hippocampus is also implicated for pathophysiology of psychiatric disorders like schizophrenia [26]. We then investigated whether Dcf1 affected social interaction (another psychiatric disorder) through the DA system.

\section{DA or a DRD1 agonist rescues social interaction deficit in NKO mice}

To test whether Dcf1 affected social interaction through the DA system, we injected DA or a DRD1 agonist into the CA1 area in NKO mice, followed by the social interaction behavioural test 7 days post-injection. We found that both DA and DRD1 agonist could rescue the social interaction deficiency of NKO mice. The injected NKO mice exhibited a clear preference for interacting with the social partner ("Stranger 1") rather than the empty cage, like the WT mice injected with saline (Fig. 3A, 3C), indicating that NKO mice displayed abnormal social interaction. When "Stranger 2" was introduced into the previously empty wire cage, mice injected with DA or the DRD1 agonist displayed a preference for the novel animal, similar to the WT mice injected with saline. NKO mice injected with saline spent more time in the chamber containing the familiar animal (Fig. 3B, 3D).

\section{DA or a DRD1 agonist could rescue the short-term plasticity deficit observed in}

\section{NKO mice}

The social interaction deficit and decreased DA may also cause electrophysiological changes. To investigate this, we used a multi-electrode array to record fEPSPs (field 
excitatory postsynaptic potentials). Compared to saline-injected WT mice, saline-injected NKO mice showed a significant decrease in the fEPSP amplitude in the CA3-CA1 Schaffer collateral pathway. Injection of DA or the DRD1 agonist increased the fEPSP amplitude in NKO mice compared with the saline-injected NKO mice (Fig. 4A).

\section{Discussion}

In this study, we provide experimental evidence that lack of Dcf1 in the nervous system leads to lower levels of DA and DRD1 and a social interaction deficit. Furthermore, we find that injection of DA or a DRD1 agonist can rescue this behavioural deficit and short-term plasticity in NKO mice. Thus, this study provides a new genetic mechanism to influence social interaction mediated by Dcf1.

We set out to investigate the higher death rate in NKO mice compared to WT and found that NKO mice displayed excessive grooming and self-injurious behaviour. We also noticed that NKO mice stayed in an empty cage rather than with other mice (Fig.1D, 1E), whereas, WT mice were always reluctant to stay in empty cages and constantly pursued new partners (Fig.1D, 1E). These social activities can lead to formation of a social network while reduced social activities, as exhibited by NKO mice, can lead to loneliness.

DA has been suggested to be the candidate neurotransmitter involved in stimulating social interaction in individuals not highly motivated to communicate [27]. Therefore, we investigated if DA was reduced upon reduced social interaction and indeed, we 
found decreased levels of DA (Fig. 2A). However, 5-HT did not decrease, although it is believed to act like DA during emotional change [23]. We thus suggest, that the role of 5-HT is far less than DA in social interaction.

The effect of DA is mediated through DA receptors, including DRD1. We demonstrated a reduction in DA and DRD1 levels associated with deficits in social activity, which is consistent with previous findings [28, 29]. We show, for the first time, that the level of DA and DRD1 decrease in Dcf1 gene knockout mice, and Dcf1 plays an important role in maintaining normal DA and DRD1 concentrations Thus, Dcf1 affects social behaviour by regulating DA and DRD1 concentrations.

Additionally, We also performed short-term plasticity experiment to investigate the relation of neuronal network to social interaction The role of several genetic regions have been previously tested using mouse models, and we found that Dcf1 affects the short-term plasticity through the DA system in CA1 area after stimulation of Schaffer collaterals.

To summarize, we found that Dcf1 controls social interaction through the DA system from the level of gene to electrophysiology. This also lays the foundation for further research involving the investigation of the mechanisms underlying this process.

\section{Material and Methods}

\section{Animals}

C57BL/6 male mice were housed at $23{ }^{\circ} \mathrm{C}$ with food and water ad libitum. All the experiments were performed in a quiet place between 9:00 a.m. and 3:00 p.m. Test mice were habituated to the testing room for at least 1 hour before the experiments. 
All animals used for the experiments were approved by the Shanghai University Ethics Committee.

\section{Production and genotyping of NKO mice}

Targeting vector in which a loxP site was inserted in the upstream regulatory sequence of Dcf1 and selecting elements of the floxed-neo gene and PGK-TK gene were inserted in the intron 1. Then Nestin-Cre mice were introduced into the Dcf1-loxp+/+ mice. Nestin was expressed on embryonic day 11, and the Cre gene in the neural stem cells expressed Cre recombinase under the action of Nestin. The Cre recombinase was able to recognize the loxP site at both ends of the Dcf1 gene to delete the Dcf1 gene in the CNS. The Nestin-Cre/Dcf1-loxP+/+ (NKO) mice were used for the experiment.

\section{HPLC}

An Agilent HPLC System was used, with a mobile phase consisting of SDS (1.4375 g/L), Na2-EDTA (0.1117 g/L), and glacial acetic acid (4.75 ml/L) diluted with ddH2O to $1 \mathrm{~L} ; 100 \mathrm{~mL}$ was used $(\mathrm{pH} \mathrm{3.9,} \mathrm{adjusted} \mathrm{with} \mathrm{glacial} \mathrm{acetic} \mathrm{acid).} \mathrm{The} \mathrm{Sample}$ Extraction Buffer consisted of $70 \%$ perchloricacid $(2.5 \mathrm{ml})$ andNa2-EDTA $(0.0134 \mathrm{~g})$ diluted with ddH2O to $200 \mathrm{ml}$. Samples from the hippocampal region were sonicated in 5 volumes $(\mathrm{w} / \mathrm{v})$ and then centrifuged for $20 \mathrm{~min}$ at $14000 \mathrm{rpm}$ at $4{ }^{\circ} \mathrm{C}$. Then, $20 \mu \mathrm{l}$ of the supernatant was used to detect the levels of DA and 5-HT in the mobile phase, with a flow rate of $1 \mathrm{ml} / \mathrm{min}$. DA and $5-\mathrm{HT}$ were identified based on the corresponding standard samples, and their retention times were determined using standard solutions $(1 \mathrm{mg} / \mathrm{ml})$.

\section{Grooming behaviour}

Young adult male mice, 3-4-months-old, were used for studying the grooming behaviour. Habituated, individually housed animals were video-taped for $24 \mathrm{~h}$ under $700 \mathrm{~lx}$ (day, $12 \mathrm{~h}$ ) and $2 \mathrm{~lx}$ (red light at night, $12 \mathrm{~h}$ ) illuminations. Grooming behaviours were coded from 19:00-19:30; grooming included all sequences of 
face-wiping, scratching of head and ears, and full-body grooming. The observer was blinded to genotype during analysis of the videotapes.

\section{Social interaction}

The social test apparatus is a box with three chambers. For the sociability test, the test animal was introduced into the middle chamber and allowed to habituate for $5 \mathrm{~min}$, after which an unfamiliar mouse (Stranger 1) was introduced into a wire cage in one of the side-chambers, leaving an empty wire cage in the other side chamber. Then, the test animal was allowed to freely explore all three chambers for $5 \mathrm{~min}$. Next, a novel stranger mouse (Stranger 2) was introduced into the wire cage that was previously empty, and the test mouse was again allowed to explore for 5 min. Social interaction. Dates were analyzed using Noldus Observer software. The observer was blinded to genotype during the scoring of the videotapes. The quantification of social behaviours was performed using Noldus Observer software by a researcher blinded to the genotype of the target animals. Quantifications included: reciprocal social interaction, as determined by any sequence or combination of sequences involving close huddling, sniffing, or allogroming by the target and stimulus mouse; the frequency of nose-to-nose sniffing; and the frequency of anogenital sniffing initiated by the target animal towards the stimulus mouse [9].

\section{Microinjection}

All surgeries were performed using a stereotaxic apparatus (Kopf Instruments; Tujunga, CA, USA) and under anaesthesia. SKF38393 hydrochloride (20 ng/ $\mu$, vol/vol, $1 \mu \mathrm{l} /$ mouse, D1 dopamine receptor partial agonist), dopamine hydrochloride $(10 \mu \mathrm{g} / \mu \mathrm{l}, \mathrm{vol} / \mathrm{vol}, 1 \mu \mathrm{l})$ or saline $(0.9 \% \mathrm{NaCl}, \mathrm{vol} / \mathrm{vol}, 1 \mu \mathrm{l})$ was injected into the CA1 area of the hippocampus. The flow rate $(0.2 \mu \mathrm{l} / \mathrm{min})$ was regulated using an 
automated syringe pump (Nanojet). All the drugs were dissolved in saline. Behavioural tests began 7 days after the surgery.

Western blot

Tissue samples were diluted in protein lysis buffer, and the SDS-PAGE gels were loaded with $10-15 \mu \mathrm{g}$ of protein per lane. The nitrocellulose membrane was blocked with $5 \%$ BSA and then incubated with primary antibodies, which included anti- $\beta$ -actin (1:2000, Santa Cruz Biotechnology) and anti-DRD1 (1:1000, Santa Cruz Biotechnology). After washing for 3 times ( 5 min each), the membrane was incubated with the secondary antibody. Then, the blot was imaged using an Odyssey system.

\section{Electrophysiology}

An MEA2100 system (Multi Channel Systems, Reutlingen, Germany) was used for examining hippocampal fEPSPs. For dissection and recording, $400 \mathrm{ml}$ of ice-cold artificial cerebrospinal fluid (ACSF; ice-water mixture) and $1 \mathrm{~L}$ of room-temperature ACSF were prepared and saturated with $95 \% \mathrm{O} 2 / 5 \% \mathrm{CO} 2$. Mice were anesthetized and decapitated, and the brains were quickly removed in the frozen ACSF and then placed in a vibratome chamber filled with ice-cold ACSF saturated with 95\% O2/5\% $\mathrm{CO}$ 2. Coronal slices $(250 \mu \mathrm{m})$ were cut at a $20^{\circ}$ incline. Slices were allowed to recover in oxygenated ACSF at room temperature for at least $2 \mathrm{~h}$. After recovery, slices were transferred to a 200/30 MEA and continuously perfused with oxygenated ACSF $(2 \mathrm{ml} / \mathrm{min})$ at $31^{\circ} \mathrm{C}$.Stimulation electrodes were placed under theCA3-CA1Schaffer collaterals in area CA1, and evoked fEPSPs were monitored at the apical dendrites of CA1 pyramidal neurons. Input/output (I/O) curves were obtained from the cortex and from area CA1 using electrical stimuli ranging from 0.5-3 V, with each stimulus step delivered three times.

\section{Statistical analysis}

The data are expressed as the mean \pm S.D. Statistical significance was analysed using Student's t-test for two groups and one-way analysis of variance (ANOVA) for more than two groups. $* \mathrm{P}<0.05, * * \mathrm{P}<0.01$, and $* * * \mathrm{P}<0.001$ wereconsidered statistically significant. 
This work was funded by the National Science Foundation of China (31070954, 81271253, 81471162), the Science and Technology Commission of Shanghai (14JC1402400), and the Key Innovation Project of Shanghai Municipal Education Commission (Grant No.14ZZ090).

\section{Conflict of interest}

The authors report no conflicts of interest.

\section{Figure Legends}

Figure 1. NKO mice exhibit deficits in social interaction.

(A) Table presenting the sex ratio and the mortality of WT and NKO mice. (B) NKO mice display neck and head lesions at 4 months of age. (C) Pre-lesion NKO mice spent more time in self-grooming than WT. (WT: $n=11$, NKO: $n=9$ ). (D) In the social interaction test, NKO mice spent less time in the chamber containing Stranger 1 (S1) and more time in the empty area (E) than WT mice. M refers to the middle chamber. (E) When analyzing social interaction by close proximity, NKO mice displayed a clear reduction in social interaction compared to WT mice. (F) In the social novelty test, NKO mice did not display a preference for the novel social partner (Stranger 2, S2), instead choosing to spend more time in the chamber with the familiar partner (Stranger 1, S1). (G) In the social novelty test, NKO mice spent significantly less time with Stranger 2. (WT: $\mathrm{n}=14$, NKO: $\mathrm{n}=13$ ). Two-tailed t-tests, ${ }^{*} P<0.05$, $* * P<0.01, * * * P<0.01 ;$ all data are presented as the mean \pm SEM.

Figure 2. NKO mice display reduced levels of DA in the hippocampus.

(A) Pictures showing the HPLC peaks of WT (up) and NKO mice (down). (B, C) The 
content of DA in the hippocampus of NKO mice was significantly lower than in WT mice (B), whereas 5-HT showed no difference (C). (D, E) DRD1 expression also showed a notable reduction in the hippocampus. Two-tailed t-tests, $* P<0.05, * * P$ $<$ 0.001; all data are presented as the mean \pm SEM; 3 mice per group.

\section{Figure 3. DA or a DRD1 agonist can rescue the social deficit in NKO mice}

(A) In the social interaction test, NKO mice injected with DA or the DRD1 agonist spent more time in the chamber containing Stranger 1 and less time in the empty area than NKO mice, showing behaviours more like the NKO mice. (B) In the social novelty test, NKO mice injected with DA or the DRD1 agonist displayed a preference for the novel social partner, like the WT mice and unlike the NKO mice. (C, D) When analyzing social interaction in close proximity, NKO mice displayed a clear reduction in social interaction compared to WT mice. One-way ANOVA was performed, $* P<$

$0.05, * * P<0.01, * * * P<0.001$; all data are presented as the mean \pm aSEM; 9 mice per group.

Figure 4. NKO mice display abnormal electrophysiology, which can be rescued by DA or a DRD1 agonist.

(A) NKO mice showed a decrease in the amplitude of evoked fEPSPs across different stimulus intensities in the hippocampus, and this deficit could be rescued by either DA or aDRD1 agonist (scale bar X: $10 \mathrm{~ms}$, Y: $0.2 \mathrm{mV}$ ). One-way ANOVA was performed 
and all data is presented as the mean \pm SEM; WT: $n=4$, NKO: $n=5, N K O+D A: n=$ 4, NKO+SKF38393: $\mathrm{n}=4 ; 3$ mice per group.

\section{References}

[1] Kringelbach ML, Rolls ET. Neural correlates of rapid reversal learning in a simple model of human social interaction. Neurolmage. 2003;20:1371-83.

[2] Sebanz N, Bekkering H, Knoblich G. Joint action: bodies and minds moving together. Trends in cognitive sciences. 2006;10:70-6.

[3] Shockley K, Richardson DC, Dale R. Conversation and coordinative structures. Topics in cognitive science. 2009;1:305-19.

[4] Kawamichi H, Sugawara SK, Hamano YH, Makita K, Kochiyama T, Sadato N. Increased frequency of social interaction is associated with enjoyment enhancement and reward system activation. Scientific reports. 2016;6:24561.

[5] Baumeister RF, Leary MR. The need to belong: desire for interpersonal attachments as a fundamental human motivation. Psychological bulletin. 1995;117:497-529.

[6] Leary MR. Commentary on self-esteem as an interpersonal monitor: The sociometer hypothesis (1995). Psychol Inq. 2003;14:270-4.

[7] House JS, Landis KR, Umberson D. Social relationships and health. Science. 1988;241:540-5.

[8] Holt-Lunstad J, Smith TB, Layton JB. Social relationships and mortality risk: a meta-analytic review. PLoS medicine. 2010;7:e1000316.

[9] Peca J, Feliciano C, Ting JT, Wang W, Wells MF, Venkatraman TN, et al. Shank3 mutant mice display autistic-like behaviours and striatal dysfunction. Nature. 2011;472:437-42.

[10] Wen T, Gu P, Chen F. Discovery of two novel functional genes from differentiation of neural stem cells in the striatum of the fetal rat. Neuroscience letters. 2002;329:101-5.

[11] Wang L, Wang J, Wu Y, Wu J, Pang S, Pan R, et al. A novel function of dcf1 during the differentiation of neural stem cells in vitro. Cellular and molecular neurobiology. 2008;28:887-94.

[12] Liu Q, Feng R, Chen Y, Luo G, Yan H, Chen L, et al. Dcf1 Triggers Dendritic Spine Formation and Facilitates Memory Acquisition. Molecular neurobiology. 2017.

[13] Farrell MR, Holland FH, Shansky RM, Brenhouse HC. Sex-specific effects of early life stress on social interaction and prefrontal cortex dendritic morphology in young rats. Behavioural brain research. 2016;310:119-25.

[14] Soares MC, Bshary R, Fusani L, Goymann W, Hau M, Hirschenhauser K, et al. Hormonal mechanisms of cooperative behaviour. Philosophical transactions of the Royal Society of London Series B, Biological sciences. 2010;365:2737-50.

[15] O'Connell LA, Hofmann HA. The vertebrate mesolimbic reward system and social behaviour network: a comparative synthesis. The Journal of comparative neurology. 2011;519:3599-639.

[16] Kim BS, Lee J, Bang M, Seo BA, Khalid A, Jung MW, et al. Differential regulation of observational fear and neural oscillations by serotonin and dopamine in the mouse anterior cingulate cortex. Psychopharmacology. 2014;231:4371-81.

[17] Nature reviews. Neuroscience. London, UK: Nature Pub. Group; 2000. p. volumes. 
[18] Cusin C, lovieno N, losifescu DV, Nierenberg AA, Fava M, Rush AJ, et al. A randomized, double-blind, placebo-controlled trial of pramipexole augmentation in treatment-resistant major depressive disorder. The Journal of clinical psychiatry. 2013;74:e636-41.

[19] Shimazu S, Minami A, Kusumoto H, Yoneda F. Antidepressant-like effects of selegiline in the forced swim test. European neuropsychopharmacology : the journal of the European College of Neuropsychopharmacology. 2005;15:563-71.

[20] Borsini F, Lecci A, Mancinelli A, D'Aranno V, Meli A. Stimulation of dopamine D-2 but not D-1 receptors reduces immobility time of rats in the forced swimming test: implication for antidepressant activity. European journal of pharmacology. 1988;148:301-7.

[21] Kalueff AV, Stewart AM, Song C, Berridge KC, Graybiel AM, Fentress JC. Neurobiology of rodent self-grooming and its value for translational neuroscience. Nature reviews Neuroscience. 2016;17:45-59.

[22] Lodge DJ, Grace AA. Aberrant hippocampal activity underlies the dopamine dysregulation in an animal model of schizophrenia. The Journal of neuroscience : the official journal of the Society for Neuroscience. 2007;27:11424-30.

[23] Kwatra M, Jangra A, Mishra M, Sharma Y, Ahmed S, Ghosh P, et al. Naringin and Sertraline Ameliorate Doxorubicin-Induced Behavioural Deficits Through Modulation of Serotonin Level and Mitochondrial Complexes Protection Pathway in Rat Hippocampus. Neurochemical research. 2016;41:2352-66.

[24] Floresco SB, West AR, Ash B, Moore H, Grace AA. Afferent modulation of dopamine neuron firing differentially regulates tonic and phasic dopamine transmission. Nature neuroscience. 2003;6:968-73.

[25] Lodge DJ, Grace AA. The hippocampus modulates dopamine neuron responsivity by regulating the intensity of phasic neuron activation. Neuropsychopharmacology : official publication of the American College of Neuropsychopharmacology. 2006;31:1356-61.

[26] Grace AA. Dopamine system dysregulation by the hippocampus: implications for the pathophysiology and treatment of schizophrenia. Neuropharmacology. 2012;62:1342-8.

[27] Straulino E, Scaravilli T, Castiello U. Dopamine depletion affects communicative intentionality in Parkinson's disease patients: Evidence from action kinematics. Cortex; a journal devoted to the study of the nervous system and behaviour. 2016;77:84-94.

[28] Straulino E, Scaravilli T, Bulgheroni M, D'Amico E, Castiello U. It's all in the type of the task: Dopamine modulates kinematic patterns during competitive vs. cooperative interaction in Parkinson's disease. Neuropsychologia. 2016;93:106-15.

[29] Homberg JR, Olivier JD, VandenBroeke M, Youn J, Ellenbroek AK, Karel P, et al. The role of the dopamine D1 receptor in social cognition: studies using a novel genetic rat model. Disease models \& mechanisms. 2016;9:1147-58. 
A

\begin{tabular}{|l|l|l|l|l|}
\hline & N & o (\%) & $\%(\%)$ & $\begin{array}{r}\text { Dead } \\
(\%)\end{array}$ \\
\hline WT & 21 & $47.59 \%$ & $52.41 \%$ & $9.52 \%$ \\
\hline NKO & 23 & $45.67 \%$ & $54.33 \%$ & $17.39 \%$ \\
\hline
\end{tabular}

B

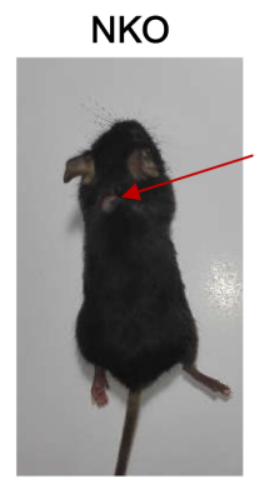

C

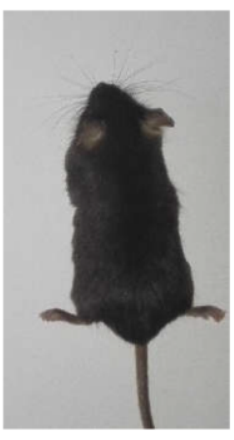

D

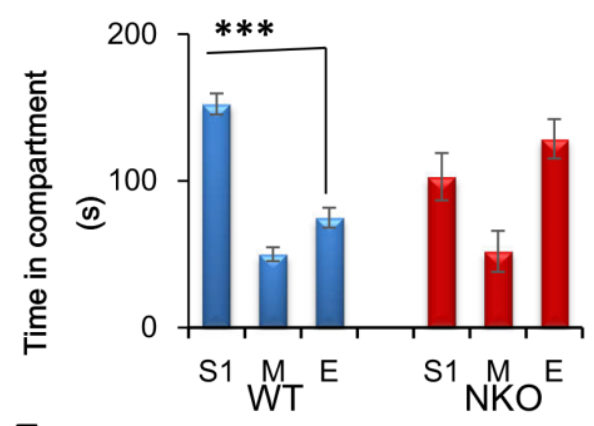

F
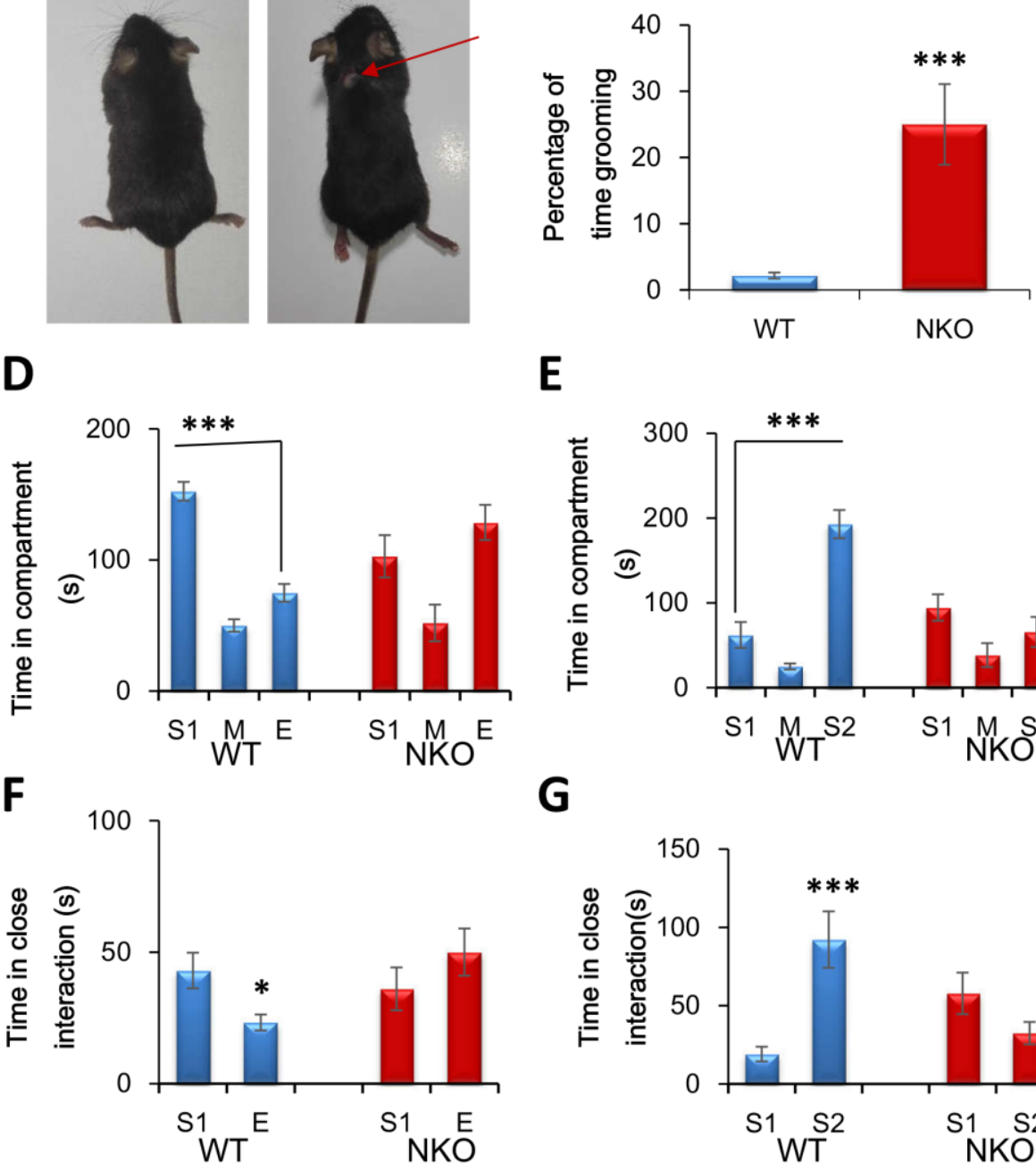

E

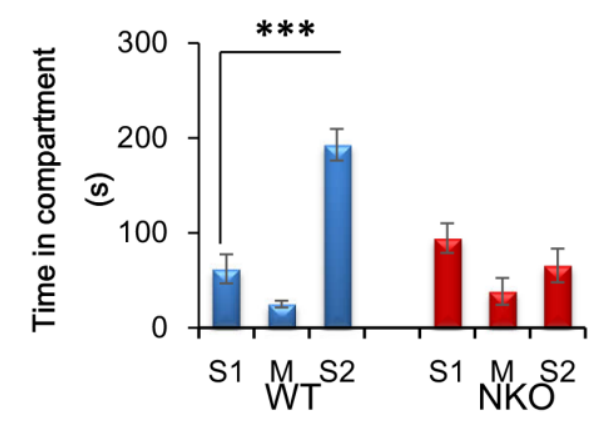

G

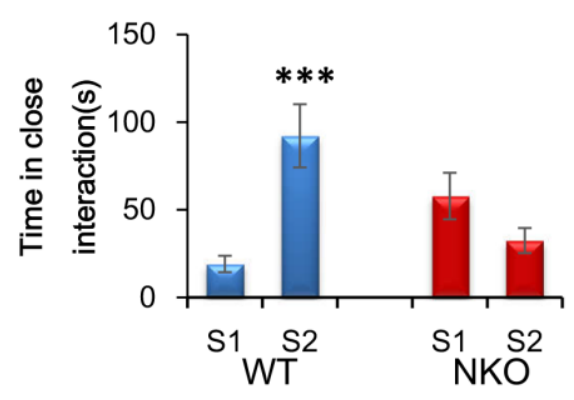


A
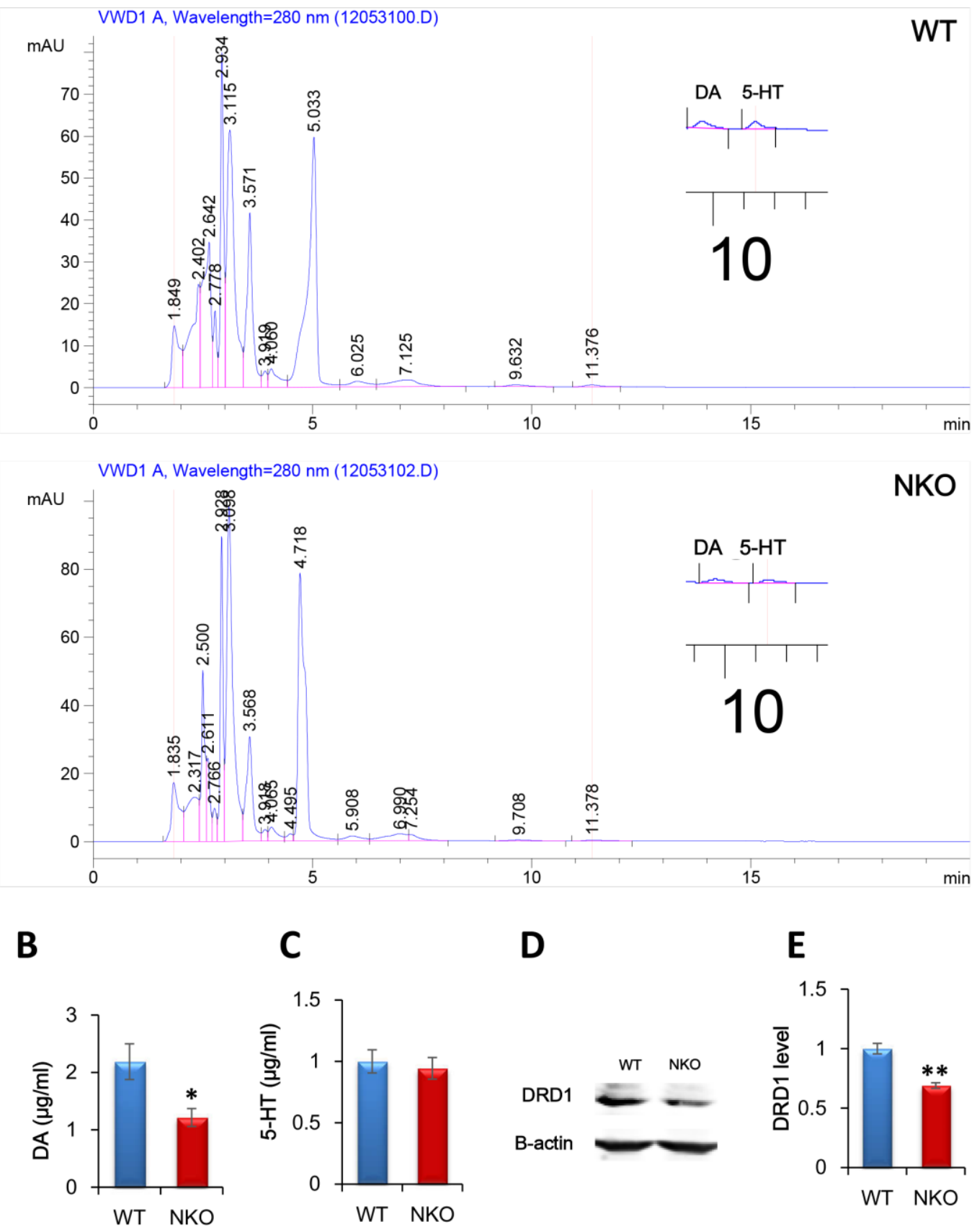
A

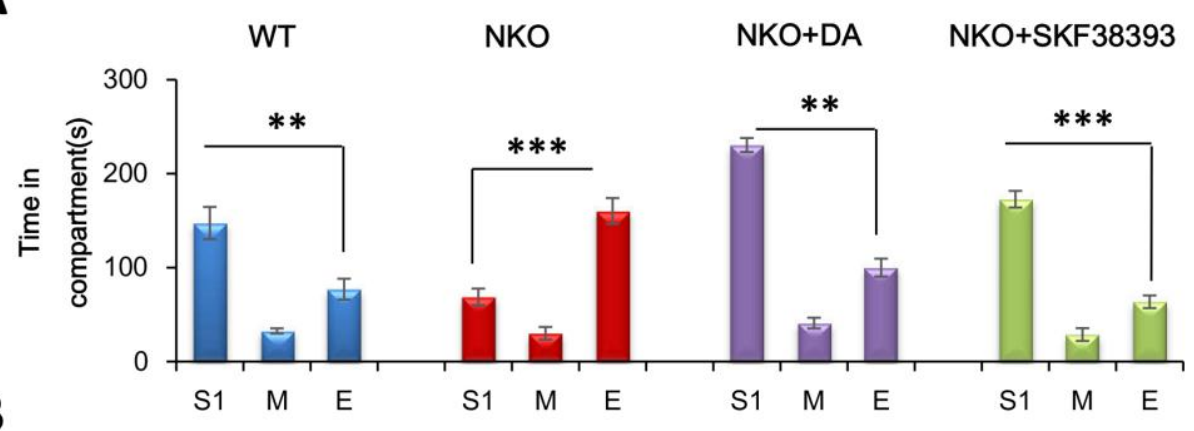

B

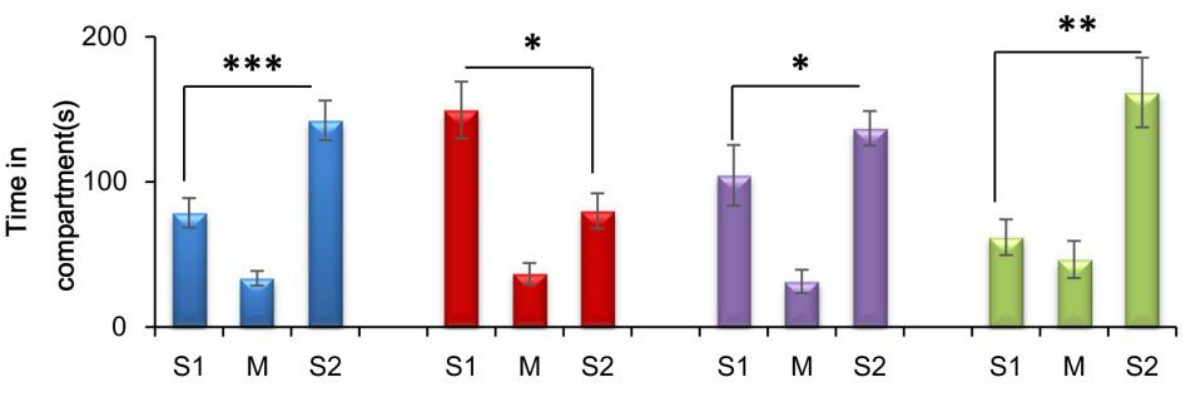

C
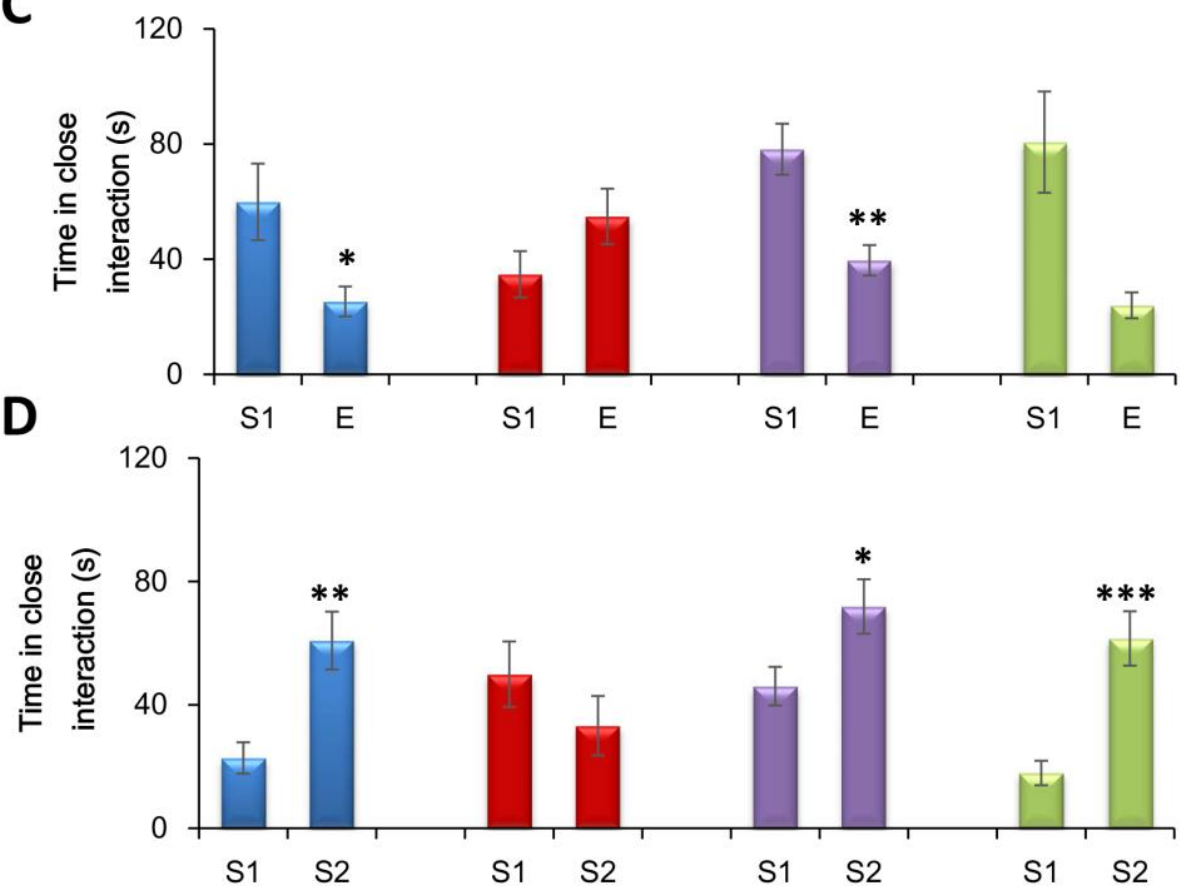
A
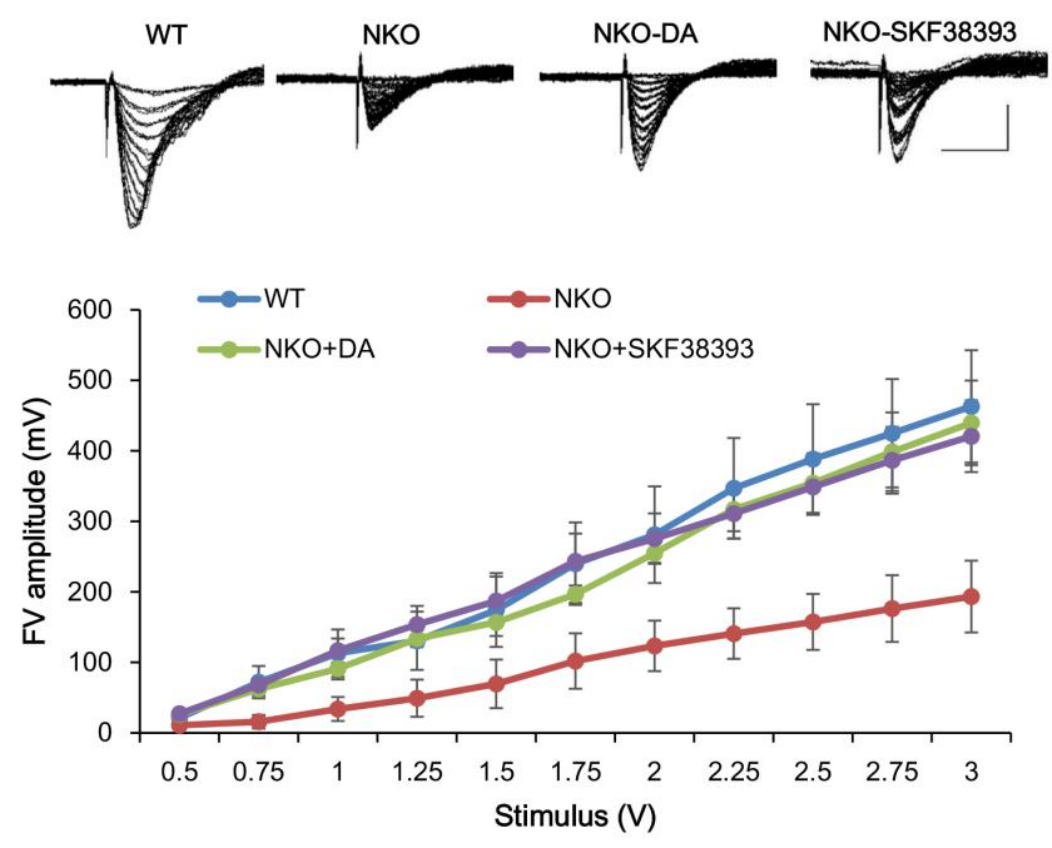